\title{
MiR-92a-3p promote s invasion and migration of hepatocellular carcinoma cells by targeting PTEN
}

\section{Yilin Hu}

Chinese PLA General Hospital

Huiling Sun

General Hospital of Central Theater Command of PLA

Qiping Lu

General Hospital of Central Theater Command of PLA

Hongliang Mei

General Hospital of Central Theater Command of PLA

Rong Liu ( $\sim$ liurong@301hospital.com.cn )

Chinese PLA General Hospital https://orcid.org/0000-0001-5170-6474

\section{Primary research}

Keywords: miR-92a-3p, hepatocellular carcinoma, PTEN, progression, CRISPR-cas12a

Posted Date: May 3rd, 2021

DOI: https://doi.org/10.21203/rs.3.rs-408247/v1

License: (c) (1) This work is licensed under a Creative Commons Attribution 4.0 International License. Read Full License 


\section{Abstract \\ Background}

MiR-92a-3p has been reported to play a part in hepatocellular carcinoma (HCC), a leading type of lethal cancer around the world. In this study, we explored the function and mechanism of miR-92a-3p in HCC.

\section{Methods}

Firstly, the expression of miR-92a-3p in HCC along with its relationship with PTEN was analyzed through biological information. To investigate the impact of miR-92a-3p on the migration and invasion of HCC cells, we performed scratch wound healing and transwell assays. Next, RT-qPCR, western blot and dual luciferase reporter gene assays were conducted to determine whether PTEN is targeted by miR-92a-3p, which was then verified through rescue assays. Afterwards, in vivo animal experiments were carried out to determine the function of miR-92a-3p in HCC tissues. As an established fact, PETN is an anti-oncogene with frequent mutation inactivation in human cancers. Thus, we used the database to predict the mutation of PETN and its mutation frequency. Finally, CRISPR-cas12a was applied to detect the R130Q mutation on PETN in HCC clinical samples.

\section{Results}

This study found that the migration and invasion of HCC could be suppressed by inhibiting miR-92a-3p, which regulates the proliferation, migration and invasion of HCC through the regulation of PETN. The bioinformatics analysis indicated higher mutation frequency of R130Q/G/L* site on the PETN gene, and greater impact of R130Q site mutation on the progression of HCC. CRISPR-cas12a detected 26 cases of R130Q mutations on PTEN in 40 HCC clinical samples

\section{Conclusion}

Collectively, this study revealed that miR-92a-3p promoted the invasion and migration of HCC by targeting PTEN, and that the stability of PETN also affected the development of HCC, which may enrich and deepen our knowledge on the progression of HCC.

\section{Introduction}

As one of the deadliest cancers around the world, hepatocellular carcinoma (HCC) accounts for above $80 \%$ of primary hepatic carcinoma globally $(1,2)$, with both incidence and mortality on the rise (3). The occurrence of HCC is mostly limited to certain regions, while its incidence rate remains the highest in East Asia, sub-Saharan Africa and Melanesia, owning to the widespread distribution of hepatitis virus (HBV) infection (4). The diagnostic methods of HCC mainly include imaging technology, blood indicators and histopathology, which, however, still lack sensitiveness and precision in the screening of $\operatorname{HCC}(5,6)$. Therefore, it is urgently needed to clarify relevant pathogenic mechanisms and ascertain possible therapeutic targets for the treatment of HCC.

MicroRNAs (miRNAs) comprise an evolutionary conserved group of small, endogenous and non-coding RNAs of about 18-25 nucleotides in length. According to report, over $60 \%$ of human protein coding genes are regulated by 
miRNAs $(7,8)$, which accounts for the downstream effects on various biological processes, e.g., the proliferation, differentiation and apoptosis of cells $(9,10)$. MiRNAs have different biological functions, and abnormally expressed miRNAs play a crucial role in a wide range of cancers, HCC included (11-13). For example, miRNA-133b suppresses tumor via negative regulation of SF3B4, which functions to promote the proliferation and metastasis of $\mathrm{HCC}$ cells (14). The study of Ni F et al. revealed the key role of miR-362-5p in the oncogenic mechanism and its promoting effect on the growth and metastasis in HCC cells by targeting CYLD (15). As an oncomiR, miR-92a-3p plays an essential role in the miR-17-92 cluster, exerting oncogenic function in a variety of cancers (16). Expressed remarkably higher in gastric cancer tissues than in adjacent non-cancerous tissues, miR-92a-3p promoted cell proliferation, migration and invasion while decreasing the apoptosis of gastric cancer cells by targeting FBXW 7 (17). By searching existing literature, we found the upregulation of miR-92a-3p (18-20) and its contribution to tumor growth in HCC (21).

Phosphatase and tensin homolog (PTEN) gene encodes phosphatase PTEN and exhibits potent anti-tumor activity. Phosphatase PTEN is involved in the dephosphorylation of membrane-bound phosphatidylserine PIP3 to PIP2, and thus regulates the PKB/AKT signaling pathway (22). In addition, PTEN is reported to play a main role in negatively regulating the PI3K/AKT pathway and inhibit PTEN-regulated apoptosis (23). It is worth noting that some reports have demonstrated the promoting effect of miR-92a-3p on proliferation, migration and invasion in esophageal squamous cell carcinoma through the regulation of PTEN (24). The study by Beng Yang et al. clarified that miR92a-3p targets PETN and regulates its downstream AKT/Snail signalling pathway, thereby contributing to the epithelial-mesenchymal transition (EMT) of receptor cancer cells (25). Importantly, PTEN is often found to be a mutated protein in many cancers (26-28), including HCC (29), and PTEN/MMAC1 gene mutation has been reported in studies (30).

This study was conducted to clarify the function of miR-92a-3p and elucidate its underlying mechanism for regulating PTEN in HCC. We began by predicting the survival of HCC patients with highly expressed PTEN and analyzing how miR-92a-3p could be correlated with PTEN in HCC. Next, in vivo and in vitro experiments were performed to investigate the role played by miR-92a-3p in the proliferation, migration and invasion of HCC. Then the targeted gene was validated through reverse transcription quantitative polymerase chain reaction (RT-qPCR), western blot and dual luciferase reporter assays. Furthermore, the differential expression of PTEN-related genes was analyzed. In addition, we applied CRISPR-cas12a technology to detect mutations in 40 clinical samples and verified the accuracy of CRISPR-cas12a with Sanger.

\section{Materials And Methods}

\section{Animal samples}

We collected female balb/c nude mice of 6 weeks old and divided them into two groups of 5 each for subcutaneous injection. With the significantly different ones removed, eventually 3 mice were selected as the experimental results. The size of tumor was measured each weak. Five weeks later, we killed all mice by cervical dissection, and collected and weighed the tumors.

\section{Cell culture}

We purchased 4 human HCC cell lines (HepG2, HepG3B, Huh7 and HCCLM3) and 1 normal liver cell line (LO2) from China Center for Type Culture Collection (Wuhan, China), which have been authenticated by STR and tested for mycoplasma contamination through Mycoplasma detection kit, and cultured them at $37^{\circ} \mathrm{C}$ under humidified 
conditions of $5 \%(\mathrm{v} / \mathrm{v}) \mathrm{CO}_{2}$ in Dulbecco's Modified Eagle's Medium (DMEM; Thermo Fisher Scientific, Waltham, MA, USA) with $10 \%$ fetal bovine serum (Gibco, Thermo Fisher Scientific, Waltham, MA, USA), 100 U/ml penicillin and $100 \mathrm{ng} / \mathrm{ml}$ streptomycin.

\section{RT-qPCR assay}

We extracted total RNA from the cells and tissues of HCC with Trizol reagent (Invitrogen, Carlsbad, CA) following the manufacturer's instructions, and then conducted reverse transcription based on the instructions of the UElris II RT-PCR System for First-Strand cDNA Synthesis (US Everbright ${ }^{\circledR} I n c$, Suzhou, China). In the RT-qPCR assay, we used SYBR Premix Ex Taq (US Everbright ${ }^{\circledR} I n c$, Suzhou, China) on an ABI 7900 system (Applied Biosystems, Foster City, CA, USA) with glyceraldehyde 3-phosphate dehydrogenase (GAPDH) adopted for endogenous control. Comparative quantification was detected using the $2^{-\triangle \Delta C t}$ method. Primers were purchased from Sangon Biotech (Shanghai, China), with sequences listed as below: GAPDH forward, 5'-GCTCTCTGC

TCCTCCTGTTC - 3'; GAPDH reverse, 5'-CGACCAAATCCGTTGACTCC-3'; PTEN forward, 5'CGGCAGCATCAAATGTTTCAG-3'; PTEN reverse, 5'-AACTGGCAGGT

AGAAGGCAACTC-3'; miR-92a-3p, 5'-UAUUGCACUUGUCCCGGCCUGU-3'; Universal primer, 5'GCGAGCACAGAATTAATACGAC-3'.

\section{Cell transfection}

Following the manufacturer's protocol, we transfected miR-92a-3p inhibitor and inhibitor negative control (NC) separately into HepG2 and HCCLM3 cells with Lipofectamine 2000 transfection reagent (Invitrogen; Thermo Fisher Scientific, Waltham, MA, USA). 48 hours after transfection, we collected the cells to perform the assays below.

\section{Cell counting kit-8 (CCK-8) assay}

HepG2 and HCCLM3 cells were separately plated into 96-well plates and cultured. About 17-24 h later, the cells were transfected with miR-92a-3p inhibitor or inhibitor NC using Lipofectamine 2000 as per the manufacturer's protocol, and then incubated for 0, 24, 48 and $72 \mathrm{~h}$. Next, CCK-8 solution (Beyotime Institute of Biotechnology, Shanghai, China) was added. After 1 hour of incubation, the absorbance at $450 \mathrm{~nm}$ was measured by Multiskan FC (Thermo Fisher Scientific, Waltham, MA, USA).

\section{Scratch wound healing assay}

HepG2 and HCCLM3 cells were plated into 6-well plates. To create uniform wounds, a $10 \mu$ tip was used for scraping before transfection (miR-92a-3p inhibitor or inhibitor NC). Exfoliated cells were removed by washing the wells thrice with phosphate-buffered saline (PBS). Then a medium containing $2 \%$ fetal bovine serum was added, and the cells were incubated in an incubator with $5 \% \mathrm{CO}_{2}$ at $37^{\circ} \mathrm{C}$, with photos magnified at $40 \times$ taken at $0,24,48$ and $72 \mathrm{~h}$ respectively.

\section{Transwell assay}

The vertical migration and invasion abilities of cells were respectively assessed using Transwell chambers uncoated or coated with Matrigel (BD Biosciences, USA). After transfection with different treatment (miR-92a-3p inhibitor, NC inhibitor, NC inhibitor + si-NC, miR-92a-3p inhibitor + si-NC and miR-92a-3p inhibitor + si-PTEN), HepG2 and HCCLM3 cells were digested to a concentration of $1 \times 10^{5} \mathrm{cells} / \mathrm{ml}$ and seeded in the upper chamber containing serum-free medium, and $600 \mu \mathrm{l}$ of $10 \%$ FBS-DMEM was added to the lower layer. After $24 \mathrm{~h}$, the upper device was 
secured, washed carefully with PBS, and fixed with $4 \%$ polyformaldehyde for 20 min at room temperature. Then the chamber was stained for 20 min in a 24-well plate with $600 \mu$ l of crystal violet, and the stained cells were observed microscopically at a magnification of $40 x$.

\section{Bioinformatics analyses}

We selected miRDB (http://mirdb.org/) and TargetScan (http://www.targetscan.org/vert_72/) to perform target gene predictions for miR-92a-3p, and detected the intersection of the obtained genes with Venny

(https://bioinfogp.cnb.csic.es/tools/venny/). Next, the STRING database (https://string-db.org/) was used for the protein-protein interactions of target genes, which were imported into Cytoscape. We obtained the top ten hub genes according to degree and calculated the enrichment modules through the Mcode in Cytoscape. Then, we conducted Kyoto Encyclopedia of Genes and Genomes (KEGG) pathway function enrichment analyses of target genes in FunRich, and performed overall survival (OS) and relapse free survival (RFS) analyses of target genes using Kaplan-Meier plotter in cBioPortal.

\section{Western blot assay}

Total proteins were extracted from HCC cells after transfection for $48 \mathrm{~h}$ using cell lysis buffer (Beyotime Biotechnology Shanghai, China). Protein samples were resolved through electrophoresis on $12 \%$ sodium dodecyl sulfate polyacrylamide gel electrophoresis (SDS-PAGE), and then transferred to polyvinylidene fluoride membrane which was blocked with $5 \%$ BSA at room temperature for $60 \mathrm{~min}$. After incubation with primary antibody PTEN (1:1000, ABclonal) at $4^{\circ} \mathrm{C}$ overnight and secondary antibody $(1: 10000$, ABclonal) at room temperature for 90 min, ECL reagents (Millpore, USA) were used to detect the signal of PTEN. We scanned the images of gels using Bio-Rad Gel Doc XR + system (Bio-Rad, Hercules, CA, USA), and adopted GAPDH as an internal control.

\section{Dual luciferase reporter gene assay}

Dual luciferase reporter gene assay corroborated the targeting relationships between PTEN and miR-92a-3p. To generate psicheck2- PTEN-WT and psicheck2-PTEN-MUT vectors, the wild type (WT) containing the predicted target site and the mutant type (MUT) with the binding site deleted were amplified and cloned into the psicheck2 plasmid. Afterwards, the luciferase vectors were respectively transfected into HEK293T cells along with miR-92a-3p inhibitor or inhibitor NC. 24 hours after transfection, following the manufacturer's protocol, we measured the relative luciferase activity by normalizing the firefly luminescence to the Renilla luminescence using the Dual-Luciferase Reporter Assay System (Promega, Madison, WI, USA).

\section{Immunohistochemistry (IHC) assay}

IHC staining was carried out to study the influence of miR-92a-3p on HCC tissues in migration and invasion. Briefly, TMA sections were washed by PBS and incubated at $37^{\circ} \mathrm{C}$ overnight with primary antibodies, 1:750 rabbit polyclonal anti-lba1 (Wako), 1:1000 anti-Mac2 (ATCC), 1:500 anti-cleaved caspase 3 (New England Biolabs), and 1:1000 rat polyclonal anti-BrdU (Axyll). The next day, TMA was washed with $0.1 \%$ Tween-20 and re-stained with DAPI. The paraffin-embedded mouse tumor sections were subjected to immunohistochemical analysis, and Ki67 and MMP9 (Proteintech, China) antibodies were used for immunohistochemical staining, respectively.

\section{LinkedOmics and DAVID analysis}

LinkedOmics (http://www.linkedomics.org/ login.php) (31) is an accessible open database with multi-omics data and clinical data on 32 cancer types and a total of 11,158 patients from The Cancer Genome Atlas (TCGA) project, offering a unique platform for biologists and clinicians to access, analyze and compare mutli-omics data within 
and across tumor types. In this study, we used LinkedOmics database to identify PTEN- associated genes and carry out gene expression correlation analyses of PTEN, and DAVID database for PTEN-associated genes Gene Ontology (GO) and KEGG pathway enrichment.

\section{cBioportal analysis}

The cBioportal for Cancer Genomics (http://cbioportal.org) (32) is an online website providing a rich diversity of resources for the exploration, visualization, and analysis of multidimensional data on cancer genomics. Since it allows survival analysis for a panel of genes, the cBioportal database was used for survival analysis. Besides, the mutation type of PTEN was also analyzed via the cBioportal database. Additionally, we used an integrated analysis via the cBioportal and ICGC(https://dcc.icgc.org/genes/)databases to identify the mutation frequency of PTEN in HCC.

\section{Detection analysis of CRISPR-cas12a Sample collection}

10-15 pieces of embedded paraffin sections were taken and store at room temperature. DNA can be extracted directly with the paraffin-embedded tissue DNA extraction kit (Tiangen Biochemical Technology Co., Ltd., item number: DP331-02).

\section{Primer design and crRNA preparation}

Wild-type and mutant templates and amplification primers were designed with the reference to the specific detection site R130Q of the gene PTEN to be detected. At the same time, crRNA was designed for these mutant regions and the required oligonucleotides (crDNA) were synthesized. crDNA and cr-T7-F were annealed to prepare the transcription templates according to the steps of double-stranded target. Then HiScribe T7 Quick High Yield RNA synthesis kit (NEB) was used without enzyme to incubate the transcriptional templates at $37^{\circ} \mathrm{C}$ for 16 hours. . After detection, 2ul DNase 1 was used to eliminate the unreacted template in the system. After purification, the required crRNA was obtained. Wild-type and mutant template sequences, amplification primers and crDNA were synthesized by Tianyi Huiyuan Company (Table 1). 
Table 1

Wild-type and mutant template sequences, isothermal amplification primers and crDNA sequences

\begin{tabular}{|ll|}
\hline Primer & sequence \\
\hline PTEN- & GCAGCAATTCACTGTAAAGCTGGAAAGGGACGAACTGGTGTAATGATATGTGCAT \\
WT & \\
PTEN- & GCAGCAATTCACTGTAAAGCTGGAAAGGGACAAACTGGTGTAATGATATGTGCAT \\
R130Q \\
MUT
\end{tabular}

\section{Verification of Crispr-cas12a fluorescence detection system}

Fncas12a was a protein to be detected. The 50-100ng template DNA with the total volume of 50 ul was added to the reagent mixture (containing $0.75 \mathrm{uM}$ crRNA, 1.5uM Fncas12a, 50pm probe and 3ul NEBuffer 3.1) And in order to read the fluorescence value and analyze the fluorescence curve, they were reacted at $37^{\circ} \mathrm{C}$ for $1 \mathrm{~h}$ in the fluorescence detector As a result, all reactions were carried out at a constant temperature of $37-42^{\circ} \mathrm{C}$.

\section{Clinical sample testing}

With 50ng DNA sample as template, RT-qPCR was performed using conventional primers. Detailed procedures are as below: samples were placed at $95^{\circ} \mathrm{C}$ for $5 \mathrm{~min}$, followed by 30 cycles of $95^{\circ} \mathrm{C}$ for $3 \mathrm{~min}, 56^{\circ} \mathrm{C}$ for $10 \mathrm{~s}, 72^{\circ} \mathrm{C}$ for $20 \mathrm{~s}$, and finally $72^{\circ} \mathrm{C}$ for $5 \mathrm{~min}$. The first-generation sequencing was performed after the electrophoresis test was qualified. Besides, 1-5ul of the amplified product was taken for Crispr-cas12a fluorescence detection.

\section{Statistical analysis}

All data in this work were presented as mean values \pm SD, with GraphPad Prism 7.0 (La Jolla, CA, USA) adopted for all statistical analyses. We used student's t test to compare two dependent groups, and two-way ANOVA with Tukey's post hoc test to compare multiple groups. Survival analyses were performed by Kaplan-Meier survival curve and determined through Log-rank test. $\mathrm{P}<0.05$ indicates a statistically significant difference.

\section{Results}




\section{The prognosis of patients with highly expressed PTEN and its relationship with miR-92a-3p}

OS and RFS of patients with HCC were depicted in Kaplan-Meier Plots. The result suggested better OS and RFS probability among patients with highly expressed PTEN (Fig. 1a and b). To analyze the relationship between PTEN and miR-92a-3p in HCC, an analysis was conducted with the StarBase v3.0 database, which demonstrated a negative correlation between the expression of PTEN and miR-92a-3p in HCC (Fig. 1c, P< 0.05).

\section{miR-92a-3p expression and prognosis analysis}

To analyze miR-92a-3p expression in different tumors, the TCGA database was employed to determine miR-92a-3p expression level. The results revealed differentially expressed miR-92a-3p in various cancers, including HCC

(Fig. 2a). Next, prognosis analysis was performed that miR-92a-3p differentially expression in HCC. As presented in Fig. $2 b$, the high expression of miR-92a-3p is correlated with much worse prognostic effect than low expression. To determine the expression level of miR-92a-3p in HCC, we selected four human HCC cell lines (HepG2, HepG3B, Huh7 and HCCLM3) and one normal liver cell line (LO2) for analysis, and RT-qPCR assays revealed higher expression of miR-92a-3p in HepG2 and HCCLM3 cell lines than in normal cells (Fig. 2c). Thus, we selected HepG2 and HCCLM3 cell lines for further research. Subsequently, HepG2 and HCCLM3 cells were respectively transfected with NC inhibitor and miR-92a-3p inhibitor, and the results demonstrated reduced miR-92a-3p expression in HepG2 and HCCLM3 cells transfected with miR-92a-3p inhibitor in comparison to those transfected with NC inhibitor (Fig. 2d).

\section{miR-92a-3p inhibitor restrains cell proliferation, migration and invasion in HCC}

To determine the role of miR-92a-3p in the proliferation, migration and invasion of HCC cells, we performed CCK-8, scratch wound healing and transwell assays in vitro. The transfection of miR-92a-3p inhibitor into HepG2 and HCCLM3 cell lines was conducted with NC inhibitor set as a control. It can be deduced from Fig. 3a and Fig. 3b that miR-92a-3p inhibitor had an obvious effect on the proliferation of HepG2 and HCCLM3 cell lines, indicating that miR-92a-3p can inhibit cell proliferation in HCC. In addition, scratch wound healing assays revealed the suppressing effect of miR-92a-3p inhibitor on the transverse migration of HepG2 (Fig. 3c) and HCCLM3 (Fig. 3d) cell lines, and transwell assays showed the repressing effect of miR-92a-3p inhibitor on the vertical migration and invasion of HepG2 (Fig. 3e) and HCCLM3 (Fig. 3f) cell lines. It can be concluded from the above findings that the migration and invasion of HCC cells can be restrained by inhibiting miR-92a-3p.

\section{Target gene validation for miR-92a-3p}

To verify PTEN as a target gene of miR-92a-3p, we performed RT-qPCR, western blot and dual luciferase reporter gene assays in vitro. MiR-92a-3p inhibitor and inhibitor NC were respectively transfected into HepG2 and HCCLM3 cells. The RT-qPCR assay demonstrated up-regulated PTEN in the miR-92a-3p inhibitor group compared to the inhibitor NC group, with up-regulation multiples of 3.98 and 3.01 in HepG2 and HCCLM3 cells, respectively (Fig. 4a). The Western blot assay suggested that the protein expression level of PTEN could be up-regulated by inhibiting miR-92a-3p (Fig. 4b). The dual luciferase reporter gene assay confirmed PTEN as a target of miR-92a-3p. With inhibitor NC as control, significantly higher relative luciferase activity was witnessed in the co-transfection of psicheck2-PTEN-WT with miR-92a-3p inhibitor (Fig. 4c, P < 0.01), while no significant difference in luciferase activity was found in the co-transfection of psicheck2-PTEN-MUT with miR-92a-3p inhibitor, suggesting the binding of miR-92a-3p to the PTEN gene. 


\section{PTEN is a target of miR-92a-3p regarding its effect on the migration and invasion of HCC}

Then, rescue and CCK-8 assays were conducted to evaluate the importance of PTEN as a target gene of miR-92a$3 p$ in the migration and invasion of HCC, and assess the impact of PTEN silencing by siRNA. Co-transfection of miR-92a-3p inhibitor and si-PTEN was conducted in HepG2 and HCCML3 cells, showing that the reducing effect of miR-92a-3p inhibitor on the migration and invasion of cell was reversed by the presence of si-PTEN (Fig. 5a and b). Rescue assays showed that these effects are reversible. Furthermore, we derived similar results from CCK-8 assays, proving that the presence of si-PTEN reversed the reducing effect of miR-92a-3p inhibitor on cell proliferation (Fig. $5 \mathrm{c}$ and d). These results revealed PTEN as a target gene of miR-92a-3p and its effect on the migration and invasion of HCC.

\section{The inhibitory effect of interfering with miR-92a-3p has been verified by in vivo experiments}

To further examine our finding in vivo, a xenograft was established which interferes with miR-92a-3p expression by injecting HepG2/miR-92a-3p inhibitor cells. According to Fig. 6a, in the HepG2/miR-92a-3p inhibitor xenograft model, the tumor size in mice with intravenous injection of miR-92a-3p inhibitor was smaller compared to the NC inhibitor control group, indicating significant inhibition of tumor growth and the remarkable tumor-inhibiting effect of miR-92a-3p inhibitor (Fig. 6b). In addition, the mean tumor weight of the miR-92a-3p inhibitor group was much less than the NC inhibitor group (Fig. 6c). Besides, western blot assays showed that PTEN expression was significantly up-regulated by knockdowned miR-92a-3p, as compared to the control group (Fig. 6d and 6e). Moreover, through immunohistochemical staining of resected tumor tissues, we found reduced positivity for Ki67 and MMP9 in tumors formed from miR-92a-3p inhibitor-transfected HepG2 cells than the NC inhibitor group (Fig. 6f). Collectively, knockdowned miR-92a-3p decreased the growth of HCC in vivo.

\section{GO and KEGG pathway inspection of co-expression genes correlated to PTEN}

We employed LinkedOmics to analyze differentially expressed genes associated with PTEN in HCC, and utilized Ttest to investigate the correlation between PTEN and genes with differential expressions in HCC (Fig. 7a). The heat map displayed the top 50 significant genes sets in positive and positive correlation to PTEN (Fig. 7b and c). Besides, significant GO and KEGG term examination by gene set enrichment analysis (GSEA) demonstrated differentially expressed genes correlated to PTEN in small cell lung cancer, prostate cancer, hepatitis B and inositol phosphate metabolism (Fig. 8).

\section{PTEN mutation status and prognosis analysis of HCC patients with PTEN mutation}

PTEN is one of the proteins with the highest mutation frequency in different cancers. To identify the mutation of PTEN in HCC, we performed an analysis via the cBioPortal database. Data shown represents analysis of cBioportal database that PTEN mutation and deep deletion were found in four studies related to HCC (Fig. 9a). Meanwhile, we also found that PTEN mutation shortens the disease-free survival of HCC patients (Fig. 9b). The results of PTEN mutation frequency analysis by cBioPortal and IGGC database showed that mutation frequency of R130Q/G/L/* sites on PTEN gene was higher, among with HCC was influenced significantly by mutation of MU29098 (R130Q) site (Fig. 9a and b). 


\section{Detection of mutations in 40 clinical samples}

To identify whether PTEN has mutations in HCC, we used CRISPR-cas12a technology to detect clinical samples for verification. The test results obtained show that 14 cases were negative non mutation samples and 26 positive heterozygous mutations were detected in 40 clinical samples (Fig. 10a). Furthermore, 40 clinical samples were repeated by Sanger sequencing to verify the accuracy of CRISPR-cas12a technology. Sanger sequencing showed that wild type coincidence rate was $93.5 \%$ and mutation coincidence rate was $100 \%$ (Fig. 10b). The detection rates of the mutated liver cancer samples by the two detection methods are $100 \%$ consistent. In addition, the CRISPRcas12a fluorescence detection system also found 4 cases of mutation samples which were not detected by Sanger sequencing. The result show in Table 2.

Table 2

CRISPR-cas12a and Sanger sequencing results

\begin{tabular}{|lll|}
\hline R130Q & $\begin{array}{l}\text { CRISPR-cas12a } \\
(\mathbf{n = 4 0})\end{array}$ & $\begin{array}{l}\text { Sanger } \\
(\mathbf{n}=\mathbf{4 0})\end{array}$ \\
\hline WT & $14(35 \%)$ & $18(45 \%)$ \\
MUT & $26(65 \%)$ & $22(55 \%)$ \\
\hline
\end{tabular}

\section{Discussion}

The occurrence and development of HCC is a complicated mechanism involving a great many molecules and signaling pathways $(33,34)$. Growing evidence indicates that miRNAs functions to either promote or suppress tumor, and plays a vital role in the initiation and progression of $\operatorname{HCC}(35,36)$. Furthermore, the abnormal expression of miR-92a-3p as a novel cancer-related miRNA has been reported in various cancers, including gastric (17), breast (16) and colorectal cancers (37). In this study, we verified the upregulation of miR-92a-3p HCC cell lines and the suppressing effect of miR-92a-3p inhibition on the migration and invasion of HCC cells in HepG2 and HCCLM3 cell lines. Notably, we found through the StarBase v3.0 database that PTEN acts as the target gene of miR-92a-3p and that PTEN is negatively correlated miR-92a-3p in HCC. It has been proved that the overexpression of miR-92a induces the EMT process by directly targeting PTEN to regulate PI3K/AKT signal activity, thus contributing to cell migration and invasion in NSCLC and tumor growth (38). Ren et al. revealed that miR-92a targeted PTEN to stimulate the growth, metastasis and chemoresistance of non-small-cell lung cancer cells (39).

With its tumor-suppressing effect recognized, the function loss of PTEN is often observed in human cancers (40). Even the slightest decrease in PTEN level and activity can contribute to cancer susceptibility and tumor progression(41), and PTEN was found to be abnormally expressed in HCC while promoting the progression of HCC $(42,43)$. In addition, there are reports on PTEN as a downstream target of various miRNAs, including miR-21(44), miR-216a/217 (45), and miR-29a (46) in HCC. The present study also proved that PTEN serves as a direct downstream target of miR-92a-3p in HCC. First, the expression of PTEN at mRNA and protein levels can be increased by transfecting miR-92a-3p inhibitor into HepG2 and HCCLM3 cells. In addition, with inhibitor NC as the control group, psicheck2-PTEN-WT co-transfected with miR-92a-3p inhibitor exhibited significantly higher relative luciferase activity. In sum, the above data indicated that the migration and invasion of HCC cells was promoted by miR-92a-3p targeting PTEN. 
PTEN polymorphisms have been associated with risks of cancer in previous studies, but none of them involves HCC. PTEN is a most frequently mutated protein in cancers. As an important tumor suppressor gene, its expression changes will affect the function of HCC, but the PTEN gene could also be inactivated by allelic deletion, gene mutation and methylation. As a contributing factor to oncogenesis, PTEN inactivation or suppression via gene mutation, aberrant subcellular localization, or altered transcriptional and post-transcriptional regulation has been found in many cancers $(47,48)$, HCC included. Therefore, the stability of PTEN itself should also be considered in this paper. Next, we studied the mutation of PTEN. By analyzing HCC samples in four studies using the cBioportal website, we noticed that in HCC, same samples had PTEN deep deletion or mutation. For this reason, it was speculated that changes in the stability of PTEN also affects tumor progression. Previous researches pointed out that the loss or mutation of PTEN may cause AKT activation and tumor progression $(49,50)$. And data also suggested GSK2636771 as a competitive selective inhibitor of PI3K $\beta$-adenosine triphosphate, as well as the possible sensitivity of cancer cells with lost PTEN function to $\beta$-isomer inhibitors that selectively target PI3K (51). PTEN has lipid phosphatase activity that functions as a direct antagonist pf PI3K-and AKT1-dependent signaling (52). Recent researches suggested that the mutation of PTEN R130Q site inactivates the phosphatidylinositol phosphatase activity of PTEN, thereby affecting its anti-tumor effect (53). In this paper, using joint cBioportal and IGGC databases, we identified that the mutation frequency of $\mathrm{R} 130 \mathrm{Q} / \mathrm{G} / \mathrm{L} /$ * is higher, and that the mutation of R130Q on PETN has a high effect on the progress of HCC. Meanwhile, the results of CRISPR-cas12a fluorescence system confirmed that $\mathrm{R} 130 \mathrm{Q}$ was a positive heterozygous mutation. There are many mutation types at PTEN R130 locus. When PTEN R130 mutation was detected in HCC clinical samples, only R130Q mutation was found. At the same time, this site was also found to be a high frequency mutation site. Later, we can further expand the sample size to detect whether it can be used as a specific target mutation site for HCC. As a new RNA-guided endonuclease, CRISPR-Cas12a has been recently recognized as an alternative genome editing tool, and gradually become powerful molecular scissors worth consideration in the application of genome editing (54). This article used CRISPR-Cas12a technology to detect mutations in clinical samples, and then Sanger to retest the accuracy and sensitivity of CRISPR-Cas12a. The test results showed that the CRISPR-Cas12a technology is more sensitive. In conclusion, our data demonstrated that miR-92a-3p played a positive role in promoting the migration and invasion of HCC via targeting PTEN, and PTEN mutation detection explored the effect of loss of PTEN's lipid phosphatase activity on HCC. This work might contribute to greater insight into the mechanism of PTEN in HCC, and provide a potential biomarker for HCC. Nonetheless, there still remain limitations despite the promising results. For instance, only bioinformatics analysis was performed for the signal pathways subject to PTEN, and experimental verification is still awaited.

\section{Abbreviations}

HCC: hepatocellular carcinoma; OS: overall survival; FPS: relapse free survival; RT-qPCR: reverse transcription quantitative polymerase chain reaction; HCC: hepatocellular carcinoma; NC: negative control; WT: wild type; MUT: mutant type; GO: gene ontology; KEGG: Kyoto Encyclopedia of Genes and Genomes.

\section{Declarations}

\section{Ethics approval and consent to participate}

Informed consent was obtained from all patients and the research was approved by the Ethics Committee of General Hospital of Central Theater Command of PLA (No. [2021]013-01). All animal experiments were performed 
with written confirmation authorized by the Animal Care and Use Committee of Hubei Provincial Center for Safety Evaluation of Food and Drug (No.202010214).

\section{Consent for publication}

Not applicable.

\section{Availability of data and materials}

The datasets used and/or analysed during the current study are available from the corresponding author on reasonable request.

\section{Competing interests}

The authors declare that they have no competing interests.

\section{Funding}

This work was supported by Hubei Province health and family planning scientific research project [grant number WJ2018H0083].

\section{Authors' contributions}

YLH and RL conceived the experiments. YLH, HLS and HLM conducted the experiments. YLH, QPL and HLM verified the underlying data. RL, HLM and HLS analyzed the results. All authors read and approved the final manuscript.

\section{Acknowledgements}

We thank Wuhan Bojie Biomedical Science and Technology CO., LTD. for the technical support and guidance provided.

\section{References}

1. El-Serag HB, Rudolph KL. Hepatocellular carcinoma: epidemiology and molecular carcinogenesis. Gastroenterology. 2007;132(7):2557-76.

2. Yang JD, Hainaut P, Gores GJ, Amadou A, Plymoth A, Roberts LR. A global view of hepatocellular carcinoma: trends, risk, prevention and management. Nature reviews Gastroenterology hepatology. 2019;16(10):589-604.

3. Njei B, Rotman Y, Ditah I, Lim JK. Emerging trends in hepatocellular carcinoma incidence and mortality. Hepatology. 2015;61(1):191-9.

4. El-Serag HB. Hepatocellular carcinoma. N Engl J Med. 2011;365(12):1118-27.

5. Dai M, Chen X, Liu X, Peng Z, Meng J, Dai S. Diagnostic Value of the Combination of Golgi Protein 73 and Alpha-Fetoprotein in Hepatocellular Carcinoma: A Meta-Analysis. PloS one. 2015;10(10):e0140067.

6. Lee J, Kim KW, Kim SY, Shin J, Park KJ, Won HJ, et al. Automatic detection method of hepatocellular carcinomas using the non-rigid registration method of multi-phase liver CT images. J X-Ray Sci Technol. 2015;23(3):275-88.

7. Cui J, Zhou B, Ross SA, Zempleni J. Nutrition, microRNAs, and Human Health. Adv Nutr. 2017;8(1):105-12. 
8. Friedman RC, Farh KK, Burge CB, Bartel DP. Most mammalian mRNAs are conserved targets of microRNAs. Genome research. 2009;19(1):92-105.

9. Hayes J, Peruzzi PP, Lawler S. MicroRNAs in cancer: biomarkers, functions and therapy. Trends Mol Med. 2014;20(8):460-9.

10. Farazi TA, Spitzer JI, Morozov P, Tuschl T. miRNAs in human cancer. J Pathol. 2011;223(2):102-15.

11. Giordano S, Columbano A. MicroRNAs: new tools for diagnosis, prognosis, and therapy in hepatocellular carcinoma? Hepatology (Baltimore. Md). 2013;57(2):840-7.

12. Reid G, Kirschner MB, van Zandwijk N. Circulating microRNAs: Association with disease and potential use as biomarkers. Crit Rev Oncol Hematol. 2011;80(2):193-208.

13. Perrotti D, Eiring AM. The new role of microRNAs in cancer. Future Oncol. 2010;6(8):1203-6.

14. Liu Z, Li W, Pang Y, Zhou Z, Liu S, Cheng K, et al. SF3B4 is regulated by microRNA-133b and promotes cell proliferation and metastasis in hepatocellular carcinoma. EBioMedicine. 2018;38:57-68.

15. Ni F, Zhao H, Cui H, Wu Z, Chen L, Hu Z, et al. MicroRNA-362-5p promotes tumor growth and metastasis by targeting CYLD in hepatocellular carcinoma. Cancer letters. 2015;356(2 Pt B):809 - 18.

16. Cun J, Yang Q. Bioinformatics-based interaction analysis of miR-92a-3p and key genes in tamoxifen-resistant breast cancer cells. 107: Biomedicine \& pharmacotherapy = Biomedecine \& pharmacotherapie; 2018. pp. 11728.

17. Zhang G, Li S, Lu J, Ge Y, Wang Q, Ma G, et al. LncRNA MT1JP functions as a ceRNA in regulating FBXW7 through competitively binding to miR-92a-3p in gastric cancer. Mol Cancer. 2018;17(1):87.

18. Wen Y, Han J, Chen J, Dong J, Xia Y, Liu J, et al. Plasma miRNAs as early biomarkers for detecting hepatocellular carcinoma. International journal of cancer. 2015;137(7):1679-90.

19. Giray BG, Emekdas G, Tezcan S, Ulger M, Serin MS, Sezgin O, et al. Profiles of serum microRNAs; miR-125b-5p and miR223-3p serve as novel biomarkers for HBV-positive hepatocellular carcinoma. Mol Biol Rep. 2014;41(7):4513-9.

20. Wang G, Dong F, Xu Z, Sharma S, Hu X, Chen D, et al. MicroRNA profile in HBV-induced infection and hepatocellular carcinoma. BMC Cancer. 2017;17(1):805.

21. Yang W, Dou C, Wang Y, Jia Y, Li C, Zheng X, et al. MicroRNA-92a contributes to tumor growth of human hepatocellular carcinoma by targeting FBXW7. Oncol Rep. 2015;34(5):2576-84.

22. Konovalov NA, Asyutin DS, Shayhaev EG, Kaprovoy SV, Timonin SY. Molecular Biomarkers of Brain and Spinal Cord Astrocytomas. Acta naturae. 2019;11(2):17-27.

23. Okahara F, Ikawa H, Kanaho Y, Maehama T. Regulation of PTEN phosphorylation and stability by a tumor suppressor candidate protein. J Biol Chem. 2004;279(44):45300-3.

24. Li X, Guo S, Min L, Guo Q, Zhang S. miR-92a-3p promotes the proliferation, migration and invasion of esophageal squamous cell cancer by regulating PTEN. Int J Mol Med. 2019;44(3):973-81.

25. Yang B, Feng X, Liu H, Tong R, Wu J, Li C, et al. High-metastatic cancer cells derived exosomal miR92a-3p promotes epithelial-mesenchymal transition and metastasis of low-metastatic cancer cells by regulating PTEN/Akt pathway in hepatocellular carcinoma. Oncogene. 2020.

26. Suzuki H, Freije D, Nusskern DR, Okami K, Cairns P, Sidransky D, et al. Interfocal heterogeneity of PTEN/MMAC1 gene alterations in multiple metastatic prostate cancer tissues. Cancer research. 1998;58(2):204-9.

27. Dahia PL. PTEN, a unique tumor suppressor gene. Endocrine-related Cancer. 2000;7(2):115-29. 
28. Ali IU, Schriml LM, Dean M. Mutational spectra of PTEN/MMAC1 gene: a tumor suppressor with lipid phosphatase activity. J Natl Cancer Inst. 1999;91(22):1922-32.

29. Shearn CT, Petersen DR. Understanding the tumor suppressor PTEN in chronic alcoholism and hepatocellular carcinoma. Adv Exp Med Biol. 2015;815:173-84.

30. Yao YJ, Ping XL, Zhang H, Chen FF, Lee PK, Ahsan H, et al. PTEN/MMAC1 mutations in hepatocellular carcinomas. Oncogene. 1999;18(20):3181-5.

31. Vasaikar SV, Straub P, Wang J, Zhang B. LinkedOmics: analyzing multi-omics data within and across 32 cancer types. Nucleic acids research. 2018;46(D1):D956-d63.

32. Gao J, Aksoy BA, Dogrusoz U, Dresdner G, Gross B, Sumer SO, et al. Integrative analysis of complex cancer genomics and clinical profiles using the cBioPortal. Sci Signal. 2013;6(269):pl1.

33. Roberts LR, Gores GJ. Hepatocellular carcinoma: molecular pathways and new therapeutic targets. Semin Liver Dis. 2005;25(2):212-25.

34. Aravalli RN, Cressman EN, Steer CJ. Cellular and molecular mechanisms of hepatocellular carcinoma: an update. Arch Toxicol. 2013;87(2):227-47.

35. Murakami Y, Yasuda T, Saigo K, Urashima T, Toyoda H, Okanoue T, et al. Comprehensive analysis of microRNA expression patterns in hepatocellular carcinoma and non-tumorous tissues. Oncogene. 2006;25(17):2537-45.

36. Jiang J, Gusev Y, Aderca I, Mettler TA, Nagorney DM, Brackett DJ, et al. Association of MicroRNA expression in hepatocellular carcinomas with hepatitis infection, cirrhosis, and patient survival. Clin Cancer Res. 2008;14(2):419-27.

37. Fu F, Jiang W, Zhou L, Chen Z. Circulating Exosomal miR-17-5p and miR-92a-3p Predict Pathologic Stage and Grade of Colorectal Cancer. Transl Oncol. 2018;11(2):221-32.

38. Jiang M, Li X, Quan X, Li X, Zhou B. MiR-92a Family: A Novel Diagnostic Biomarker and Potential Therapeutic Target in Human Cancers. Frontiers in molecular biosciences. 2019;6:98.

39. Ren P, Gong F, Zhang Y, Jiang J, Zhang H. MicroRNA-92a promotes growth, metastasis, and chemoresistance in non-small cell lung cancer cells by targeting PTEN. Tumour biology: the journal of the International Society for Oncodevelopmental Biology Medicine. 2016;37(3):3215-25.

40. Song MS, Salmena L, Pandolfi PP. The functions and regulation of the PTEN tumour suppressor. Nat Rev Mol Cell Biol. 2012;13(5):283-96.

41. Lee YR, Chen M, Pandolfi PP. The functions and regulation of the PTEN tumour suppressor: new modes and prospects. Nat Rev Mol Cell Biol. 2018;19(9):547-62.

42. Meng F, Henson R, Wehbe-Janek H, Ghoshal K, Jacob ST, Patel T. MicroRNA-21 regulates expression of the PTEN tumor suppressor gene in human hepatocellular cancer. Gastroenterology. 2007;133(2):647-58.

43. Wan XW, Jiang M, Cao HF, He YQ, Liu SQ, Qiu XH, et al. The alteration of PTEN tumor suppressor expression and its association with the histopathological features of human primary hepatocellular carcinoma. J Cancer Res Clin Oncol. 2003;129(2):100-6.

44. Bao L, Yan Y, Xu C, Ji W, Shen S, Xu G, et al. MicroRNA-21 suppresses PTEN and hSulf-1 expression and promotes hepatocellular carcinoma progression through AKT/ERK pathways. Cancer letters. 2013;337(2):22636.

45. Xia H, Ooi LL, Hui KM. MicroRNA-216a/217-induced epithelial-mesenchymal transition targets PTEN and SMAD7 to promote drug resistance and recurrence of liver cancer. Hepatology. 2013;58(2):629-41. 
46. Kong G, Zhang J, Zhang S, Shan C, Ye L, Zhang X. Upregulated microRNA-29a by hepatitis B virus X protein enhances hepatoma cell migration by targeting PTEN in cell culture model. PloS one. 2011;6(5):e19518.

47. Steck PA, Pershouse MA, Jasser SA, Yung WK, Lin H, Ligon AH, et al. Identification of a candidate tumour suppressor gene, MMAC1, at chromosome 10q23.3 that is mutated in multiple advanced cancers. Nat Genet. 1997;15(4):356-62.

48. Di Cristofano A, Pandolfi PP. The multiple roles of PTEN in tumor suppression. Cell. 2000;100(4):387-90.

49. Duvoux C, Toso C. mTOR inhibitor therapy: Does it prevent HCC recurrence after liver transplantation? Transplantation reviews (Orlando. Fla). 2015;29(3):168-74.

50. Nakanishi A, Wada Y, Kitagishi Y, Matsuda S. Link between PI3K/AKT/PTEN Pathway and NOX Proteinin Diseases. Aging disease. 2014;5(3):203-11.

51. Mateo J, Ganji G, Lemech C, Burris HA, Han SW, Swales K, et al. A First-Time-in-Human Study of GSK2636771, a Phosphoinositide 3 Kinase Beta-Selective Inhibitor, in Patients with Advanced Solid Tumors. Clinical cancer research: an official journal of the American Association for Cancer Research. 2017;23(19):5981-92.

52. Lu YM, Cheng F, Teng LS. The association between phosphatase and tensin homolog hypermethylation and patients with breast cancer, a meta-analysis and literature review. Scientific reports. 2016;6:32723.

53. Han SY, Kato H, Kato S, Suzuki T, Shibata H, Ishii S, et al. Functional evaluation of PTEN missense mutations using in vitro phosphoinositide phosphatase assay. Cancer research. 2000;60(12):3147-51.

54. Chen JS, Ma E, Harrington LB, Da Costa M, Tian X, Palefsky JM, et al. CRISPR-Cas12a target binding unleashes indiscriminate single-stranded DNase activity. Science (New York). 2018;360(6387):pp. 436-9.

\section{Figures}

a

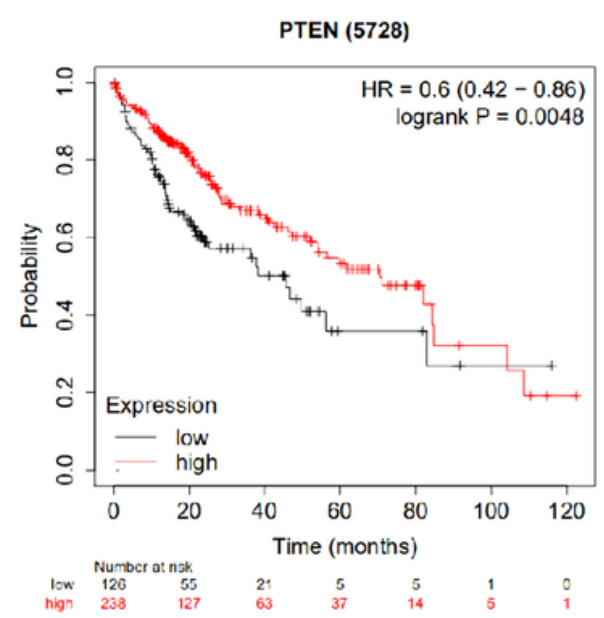

b

RFS:Relapse-Free Survival

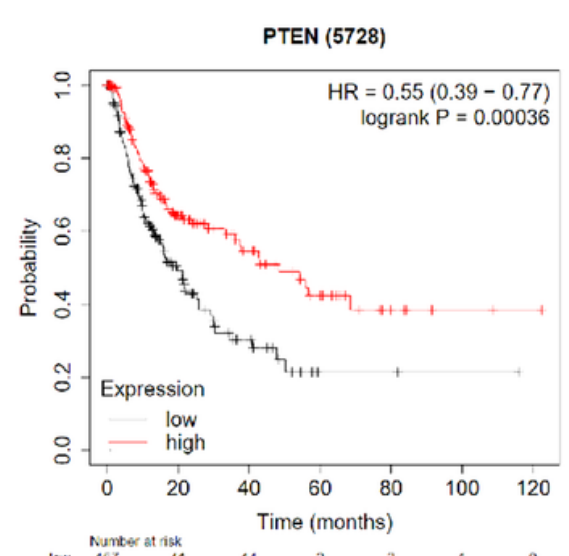

C

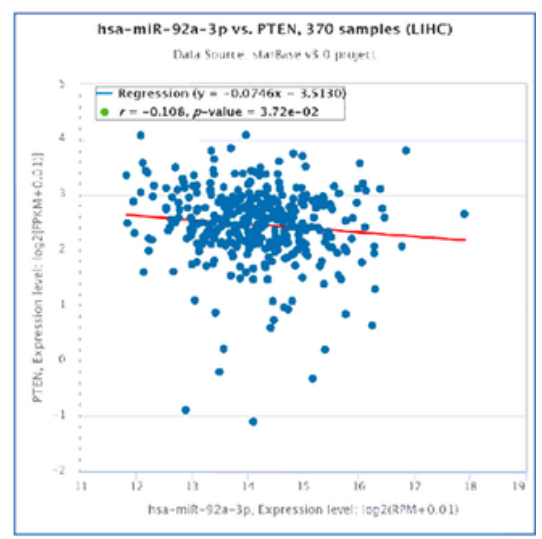

\section{Figure 1}

Prognosis and correlation analysis. Notes: (a) Kaplan-Meier survival curve shows that patients with high PTEN expression have a nice OS. (b) Kaplan-Meier survival curve shows that patients with high PTEN expression have a nice RFS. (c) The negative correlation between PTEN and miR-92a-3p was analyzed by the StarBase v3.0 online database. ${ }^{*} \mathrm{P}<0.05$. Abbreviations: HCC, hepatocellular carcinoma; OS, overall survival; FPS, relapse free survival. 

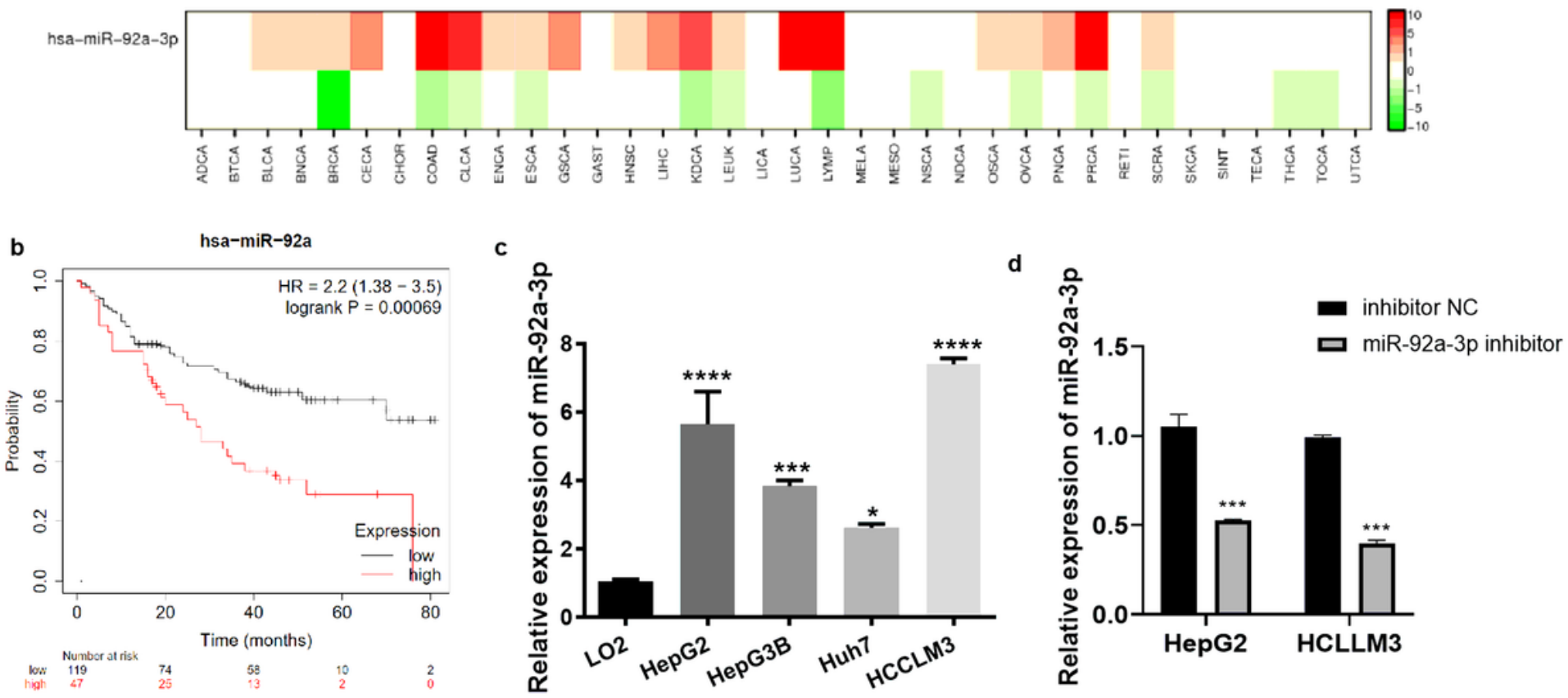

Figure 2

Expression of miR-92a-3p and its prognosis analysis. Notes: (a) miR-92a-3p is differentially expressed in a variety of tumor cells. (b) Kaplan-Meier survival curve shows that patients with high miR-92a-3p expression have a worse survival rate on HCC. (c) The expression levels of miR-92a-3p in normal and four HCC cell lines as determined by RT-qPCR assays. (d) Inhibitor miR-92a-3p into HepG2 and HCLLM3 cells. The values are presented as the mean \pm SD. Two-way ANOVA test used to analyze differences between two groups. * $P<0.001$. Abbreviations: RT-qPCR, reverse transcription quantitative polymerase chain reaction; HCC, hepatocellular carcinoma. 
a HepG2

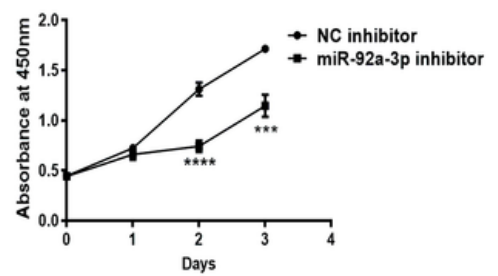

C
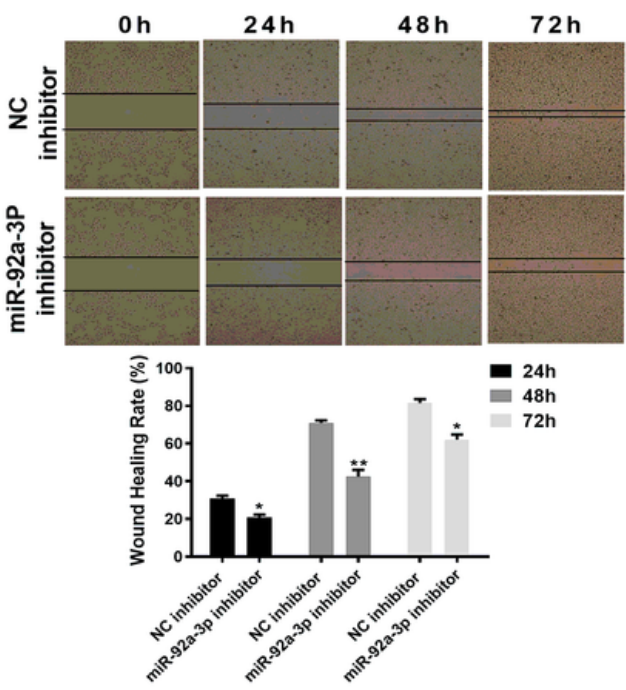

e

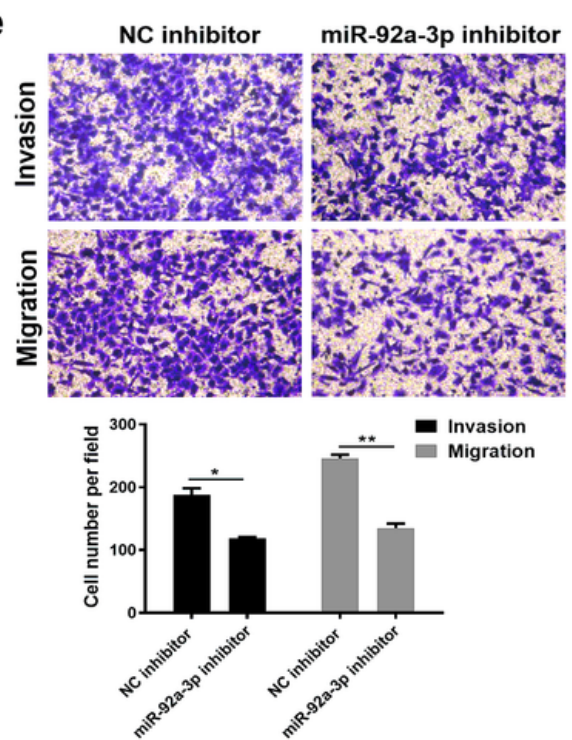

b HCCLM3

d

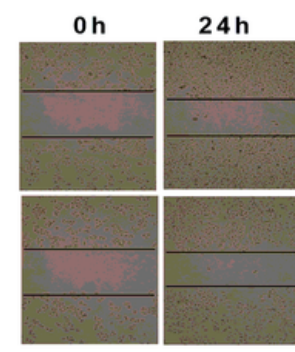

$48 \mathrm{~h}$ $72 \mathrm{~h}$
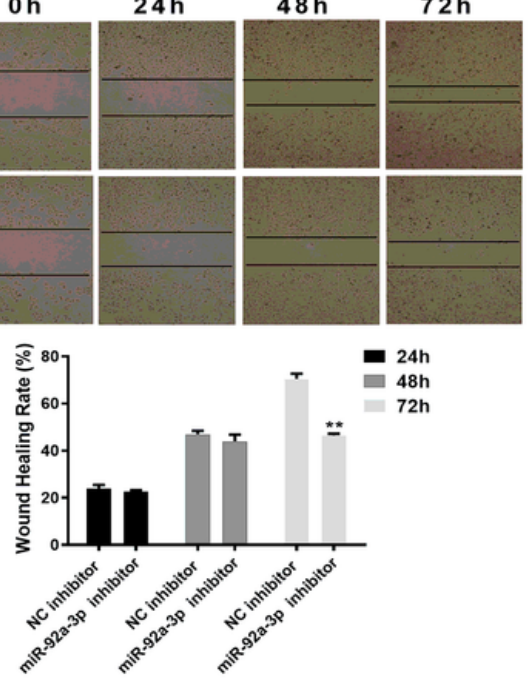

f
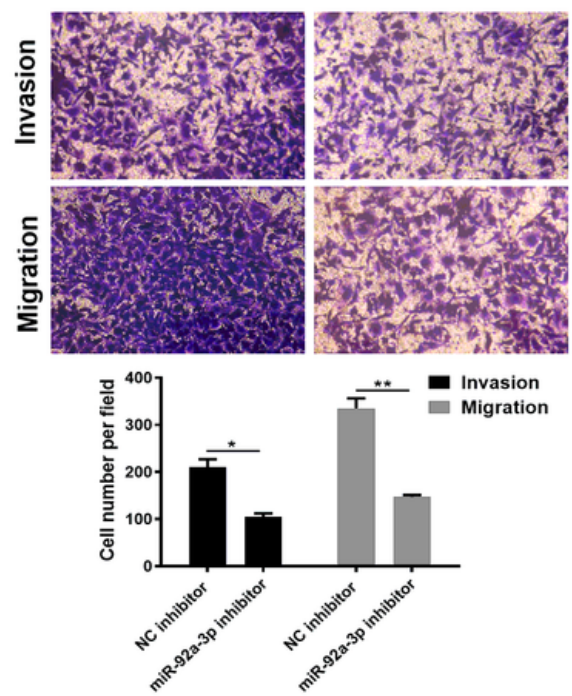

Figure 3

miR-92a-3p inhibitor restrains proliferation, migration and invasion of HCC cells. Notes: The proliferation of cells transfected with NC inhibitor and miR-92a-3p inhibitor as determined by CCK-8 assays in (a) HepG2 and (b) HCCLM3 cell lines. The transverse migration of cells transfected with inhibitor NC and miR-92a-3p inhibitor as determined by scratch wound healing assays in (c) HepG2 and (d) HCCLM3 cell lines at a magnification of 40x. The invasion and vertical migration of cells transfected with inhibitor NC and miR-92a-3p inhibitor as determined by Transwell assays in (e) HepG2 and (f) HCCLM3 cell lines. *, $\mathrm{P}<0.05$; **, $\mathrm{P}<0.01$. Abbreviations: HCC, hepatocellular carcinoma; NC, negative control. 
a

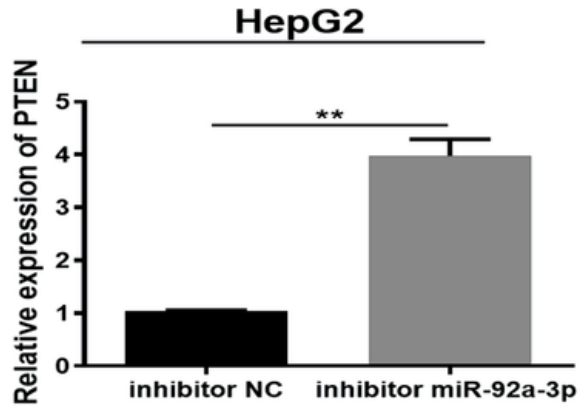

b
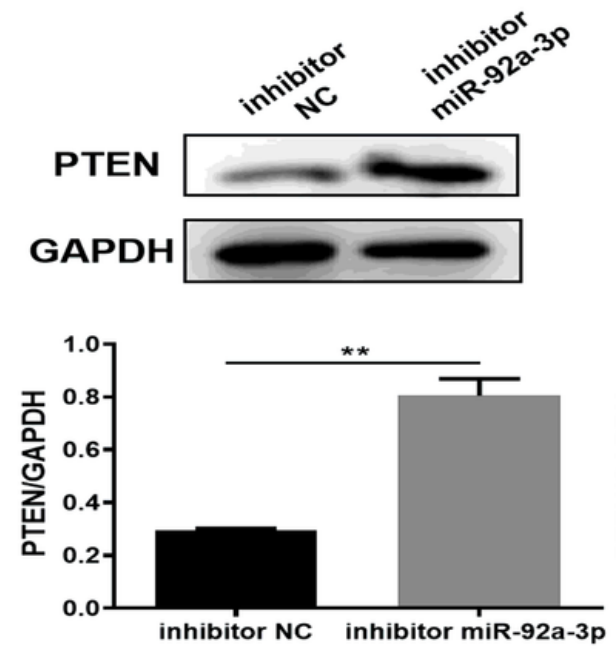

C
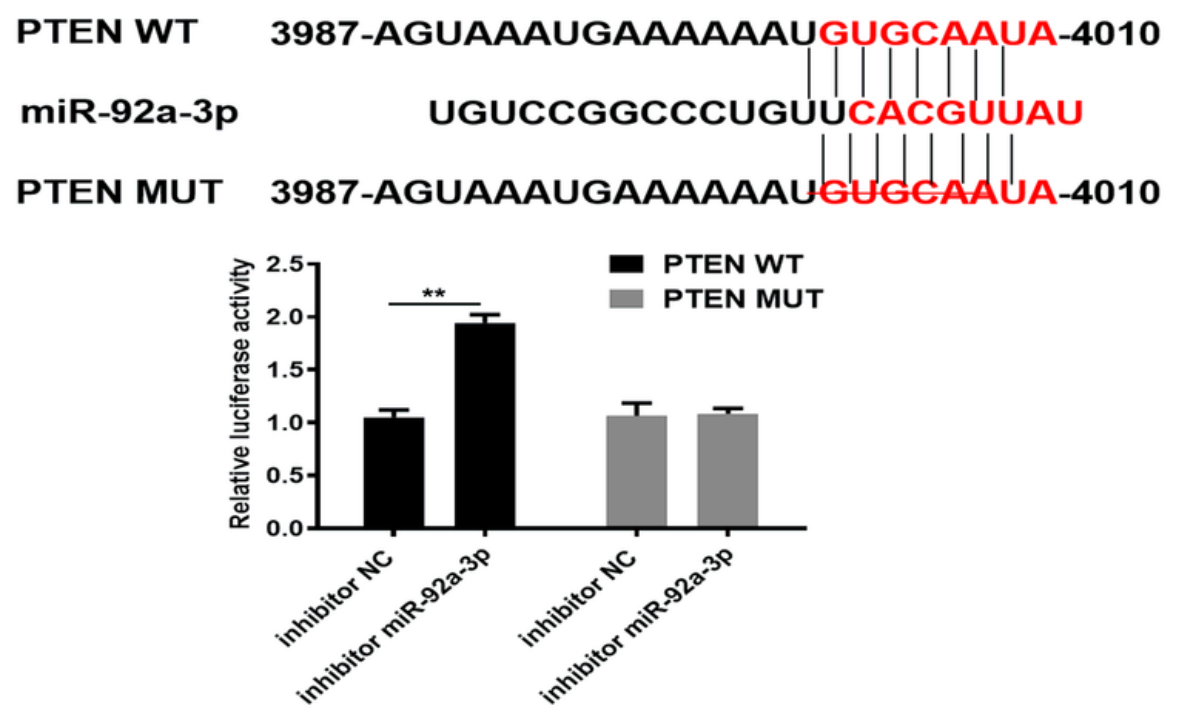

HCCLM3
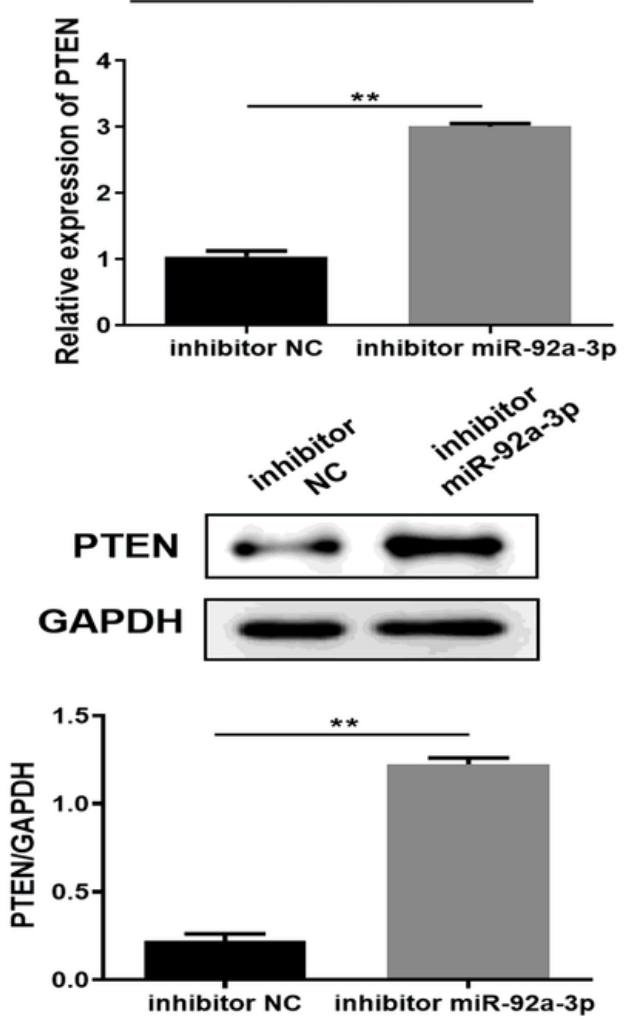

Figure 4

Target gene validation of miR-92a-3p. Notes: Relative expression of PTEN as analyzed by (a) RT-qPCR assays and (b) western blot assays in cells transfected with inhibitor NC and miR-92a-3p inhibitor in HepG2 and HCCLM3 cell lines. (c) Effects of miR-92a-3p on the luciferase reporter activity of cells with PTEN WT or PTEN MUT.

Abbreviations: WT, wild type; MUT, mutant type; NC, negative control; RT-qPCR, reverse transcription quantitative polymerase chain reaction. 

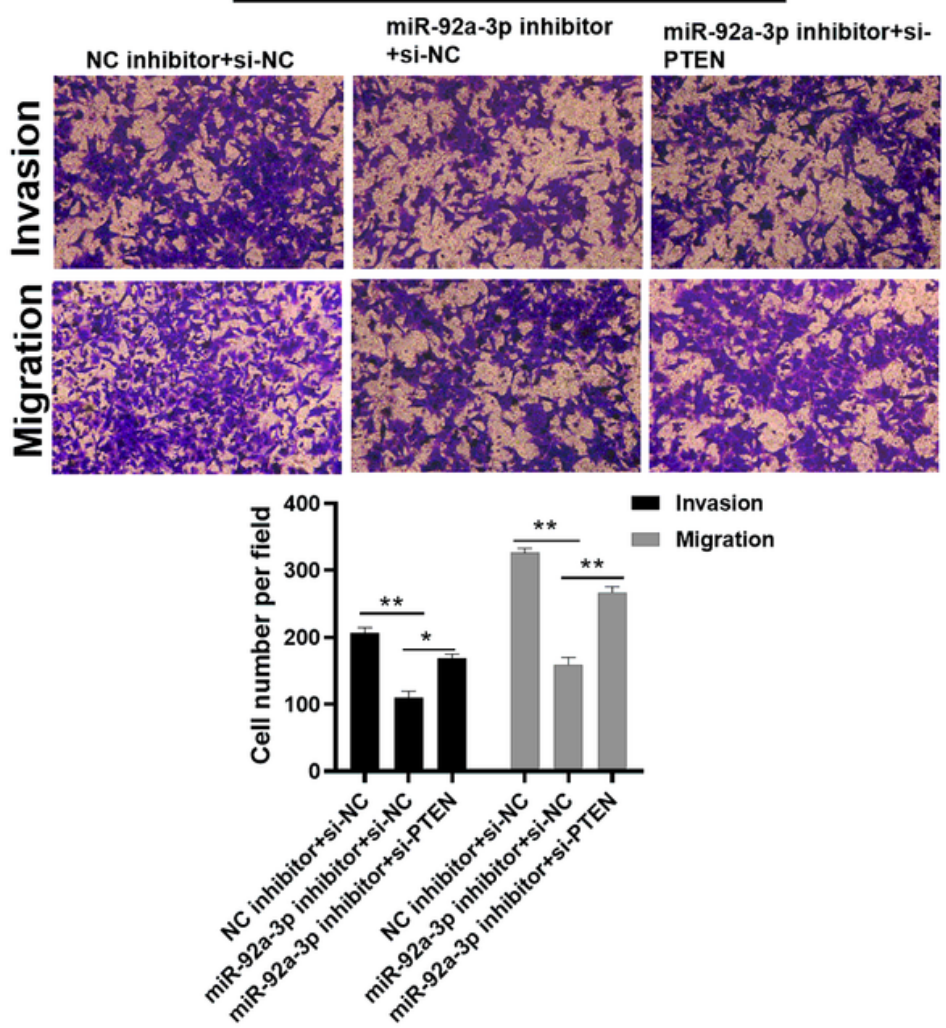

C

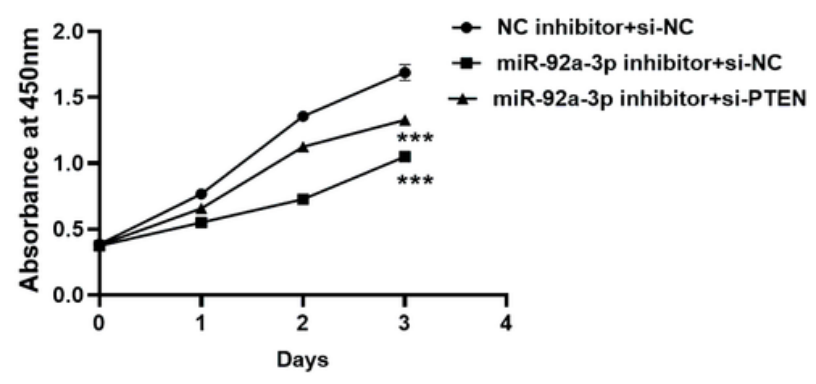

b

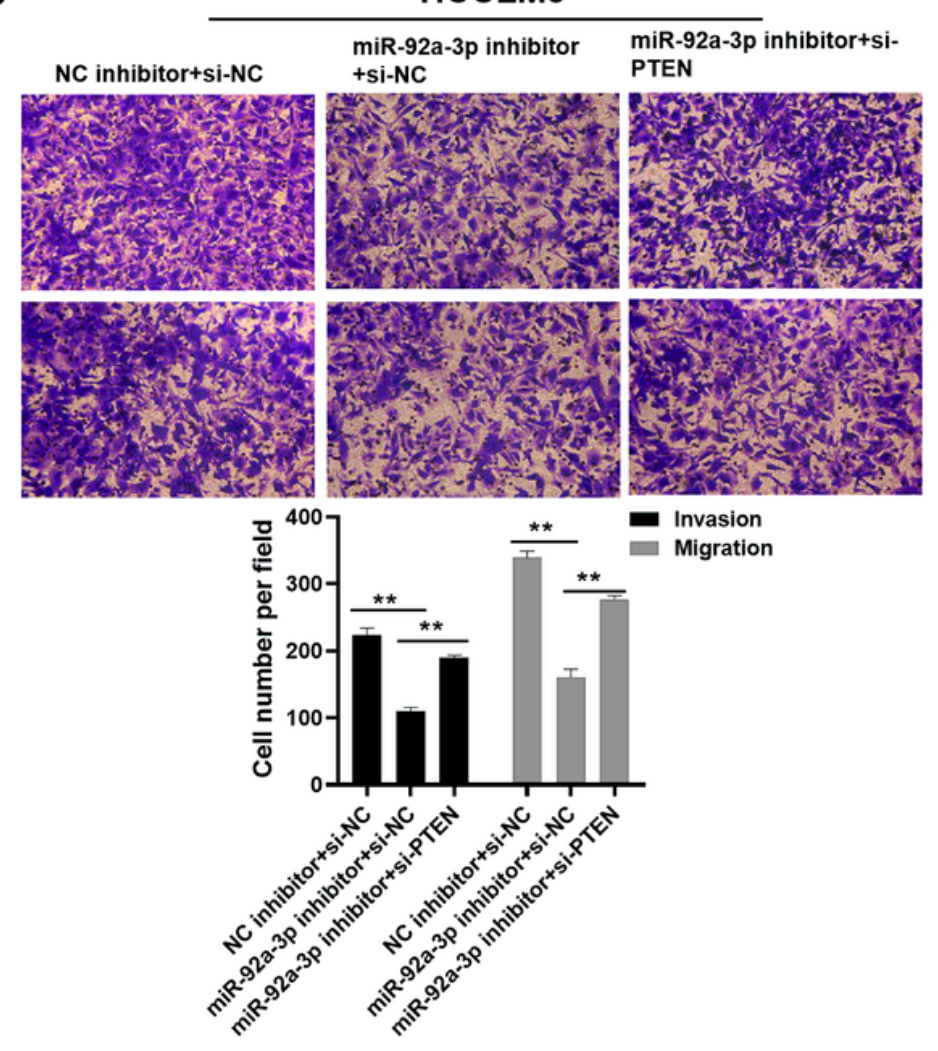

d

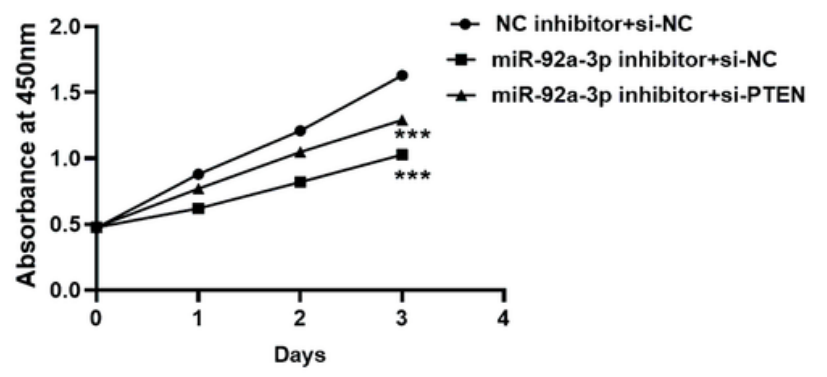

Figure 5

Silencing PTEN reverses the migration and invasion suppressing effect of miR-92a-3p inhibitor in HCC. The invasion and vertical migration of cells transfected with inhibitor NC+si-NC, miR-92a inhibitor + si-NC and miR-92a inhibitor+si-PTEN as determined by Transwell assays in (a) HepG2 and (b) HCCLM3 cell lines. *, $P<0.05 ; * \star, P<0.01$. The proliferation of cells transfected with inhibitor NC+si-NC, miR-92a inhibitor+si-NC and miR-92a inhibitor+siPTEN as determined by CCK-8 assays in (c) HepG2 and (d) HCCLM3 cell lines. 
a

\section{NC inihibitor \\ miR-92a inihibitor}

C

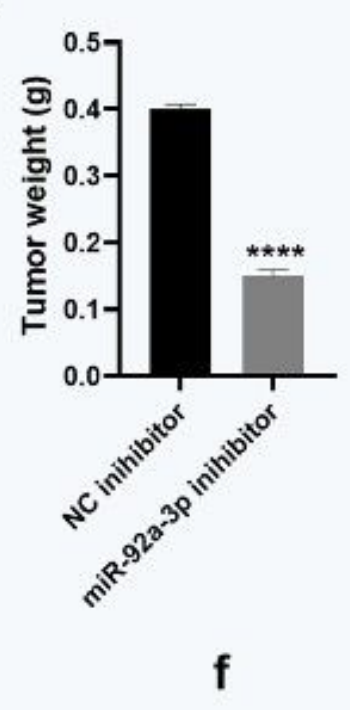

Ki67

MMP9

d

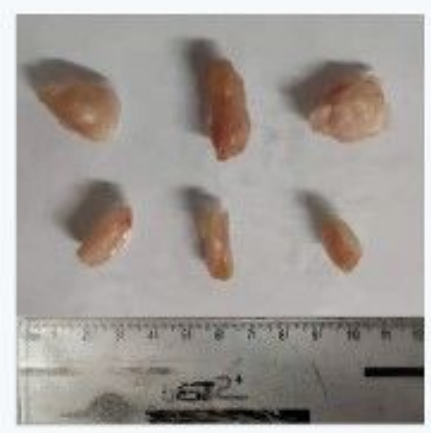

b

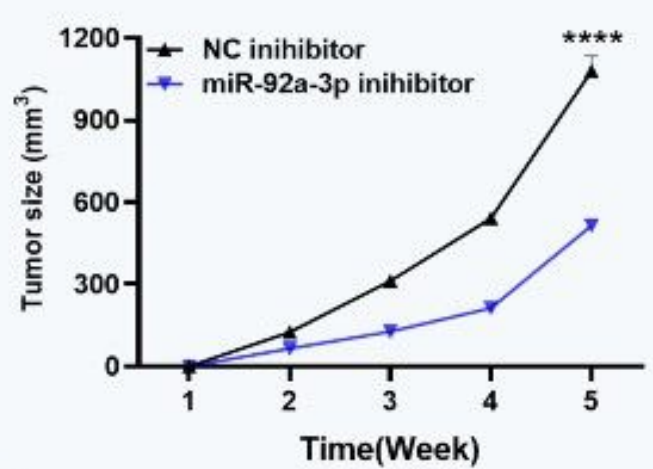

e
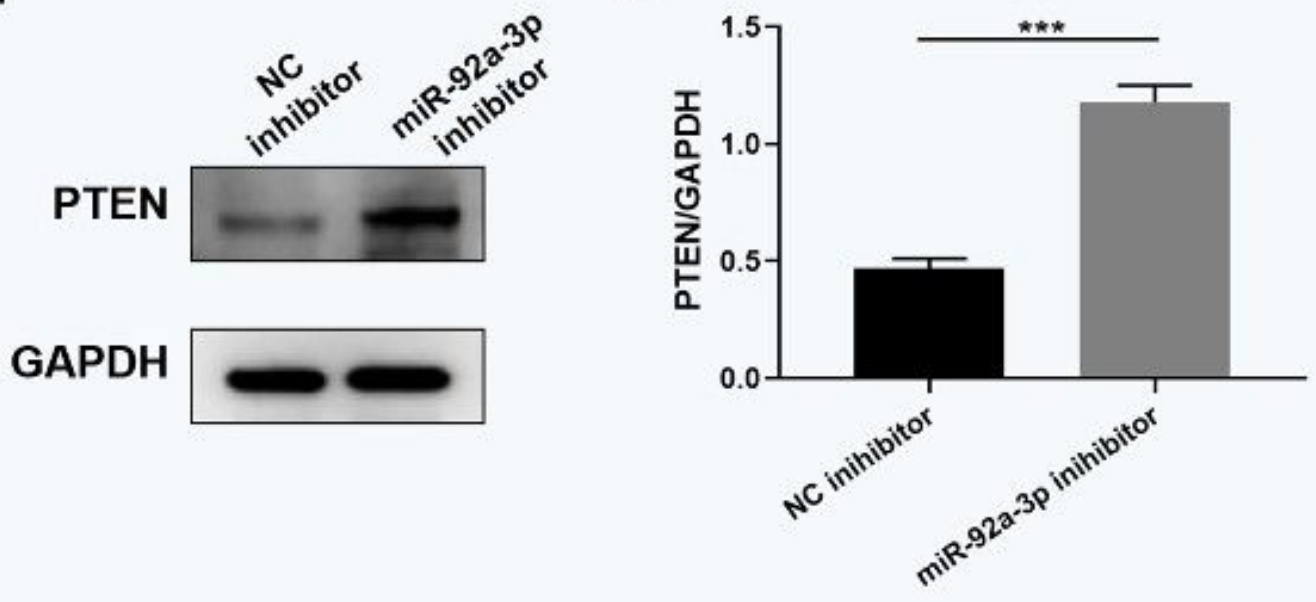

NC inihibitor

miR-92a inihibitor

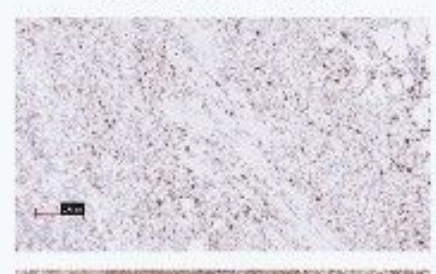

in
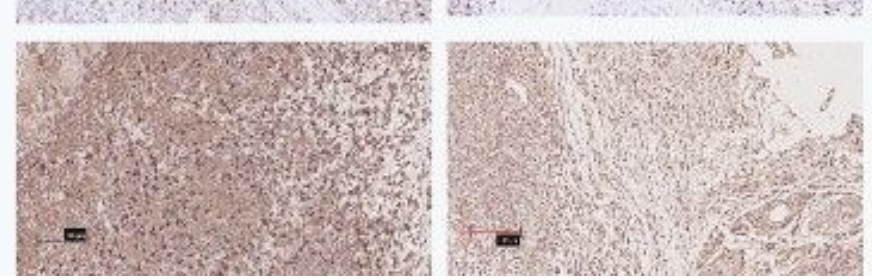

Figure 6

miR-92a suppresses tumor growth in vivo. (a) Representative tumors in each group are shown. (b) The tumor volume was examined every 7 days after the first injection. $* \star * P<0.001$. (c) The tumor weight was analyzed at 5 weeks after the first injection. ( $d$ and e) The protein expression level of PTEN was determined by using western blot assays. (f) The tumor tissue samples of the designated group were stained, and the levels of Ki67 and MMP9 protein were detected by immunohistochemistry. 


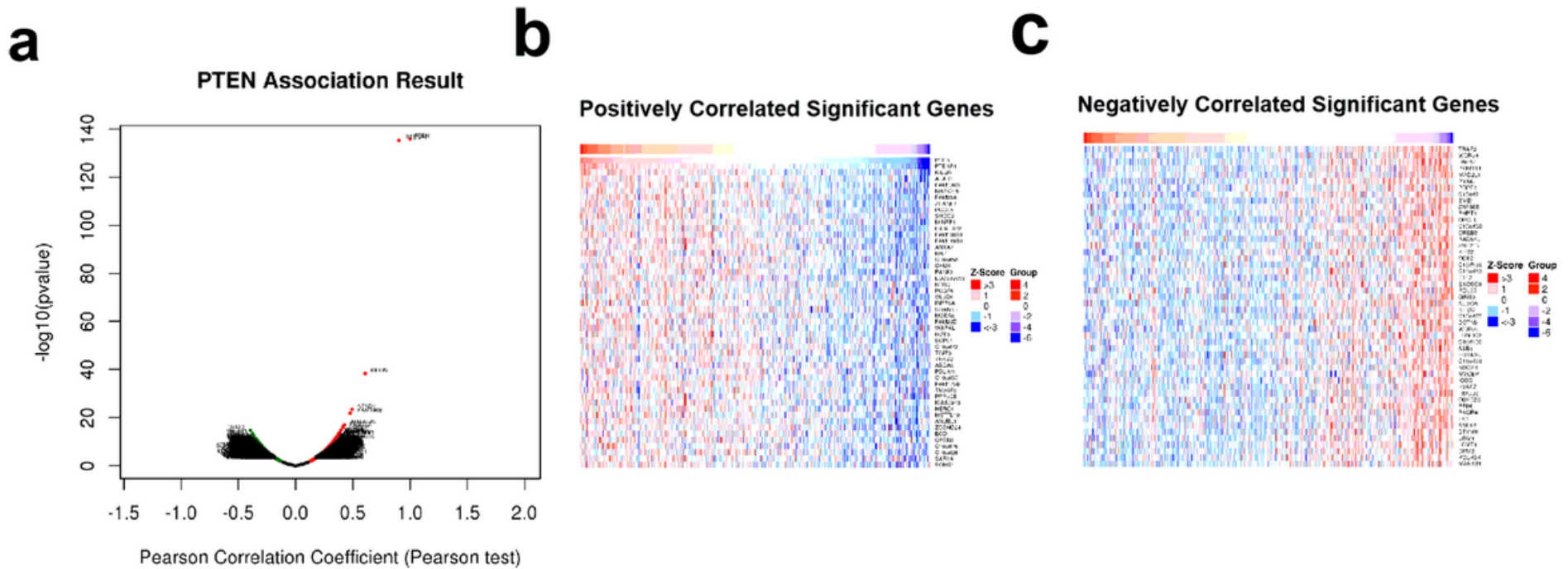

\section{Figure 7}

Genes differentially expressed in association with PTEN in HCC according to LinkedOmics. Notes: (a) The correlation between PTEN and genes differentially expressed in HCC was evaluated by Pearson test. (b) Heat map of differentially expressed genes showed the top-50 significant genes positively and (c) negatively correlated with PTEN in HCC. The red stands for positively correlated genes and the blue stands for negatively correlated genes. Abbreviations: HCC, hepatocellular carcinoma.

\section{Positively Correlated Significant Genes}

$\mathbf{a}$

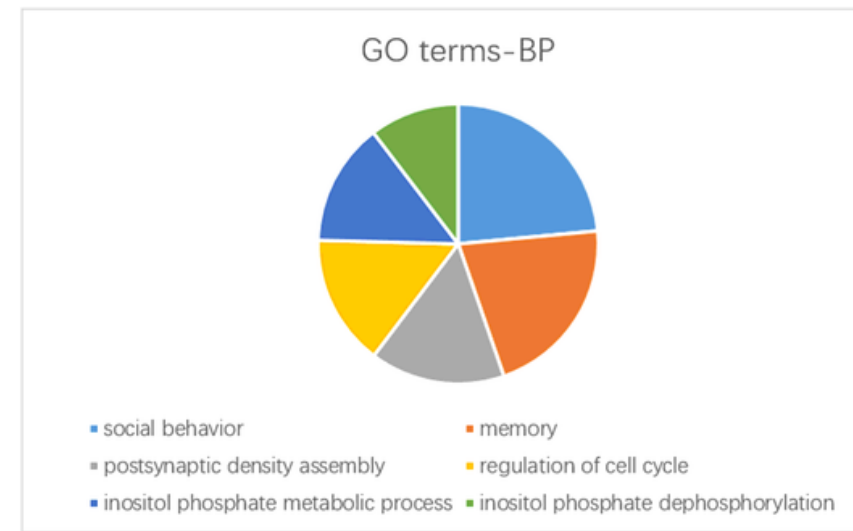

C

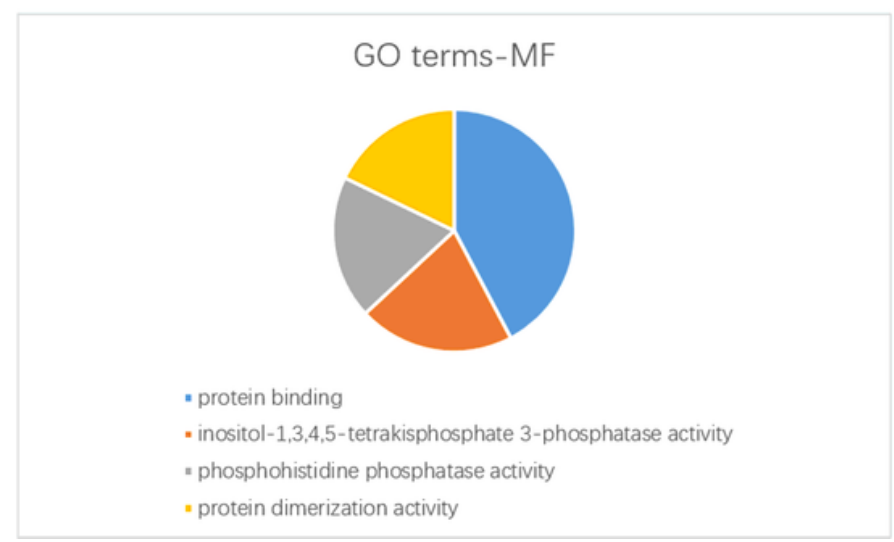

b

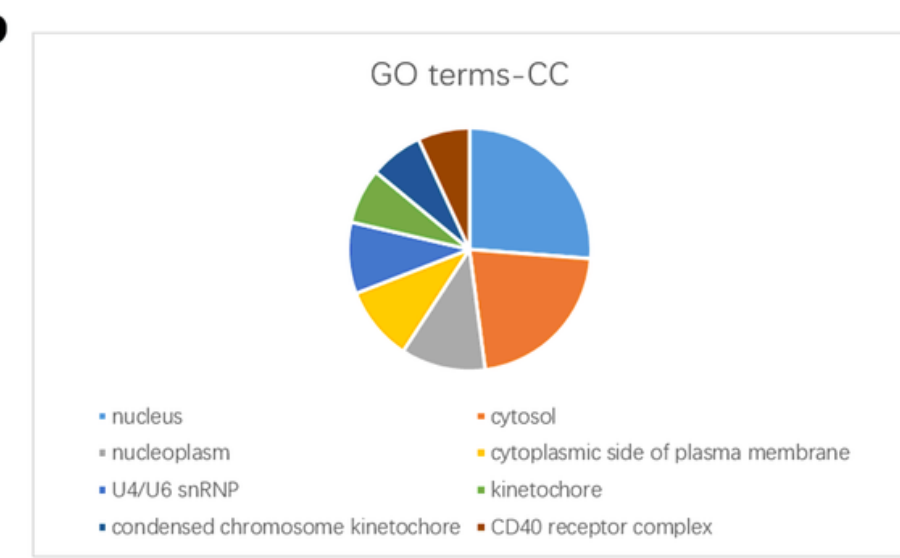

d

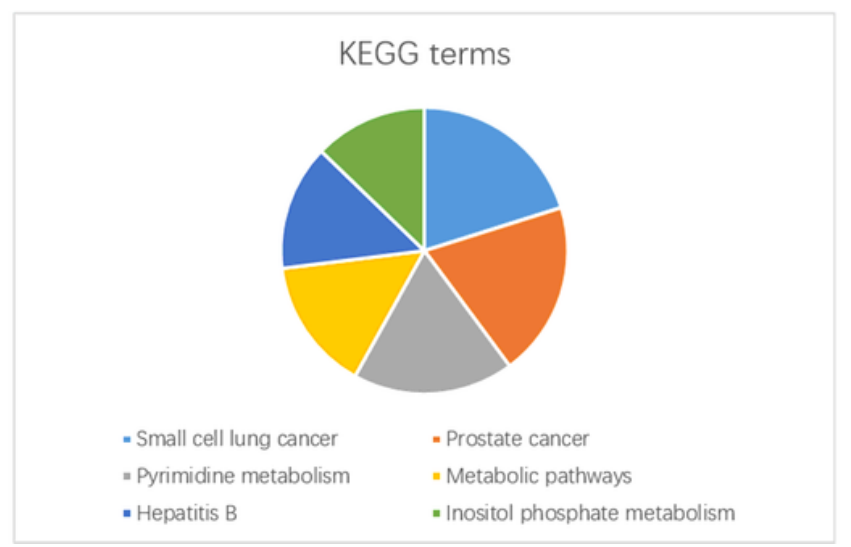

Figure 8 
Significantly enriched GO and KEGG pathways of PTEN in HCC. Notes: The significantly enriched GO and KEGG pathways of PTEN co-expression genes in HCC were analyzed. (a) KEGG pathway analysis. (b) Biological processes. (c) Cellular components. (d) molecular functions. Abbreviations: HCC, hepatocellular carcinoma; GO, gene ontology; KEGG, Kyoto Encyclopedia of Genes and Genomes.

a

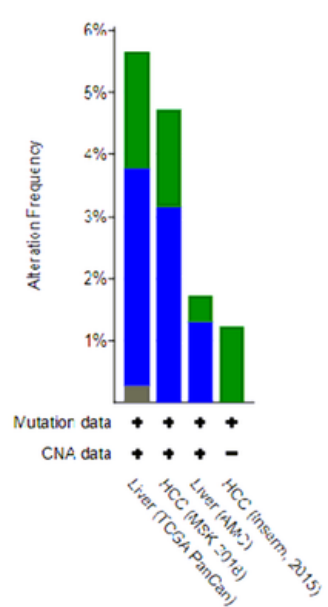

b

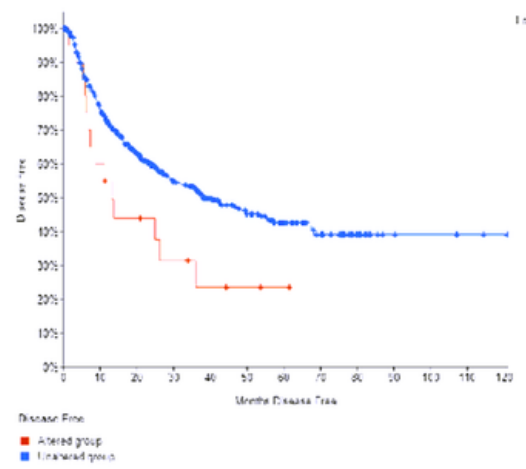

- Mutation

- Deep Deletion

- Multiple Alterations

C

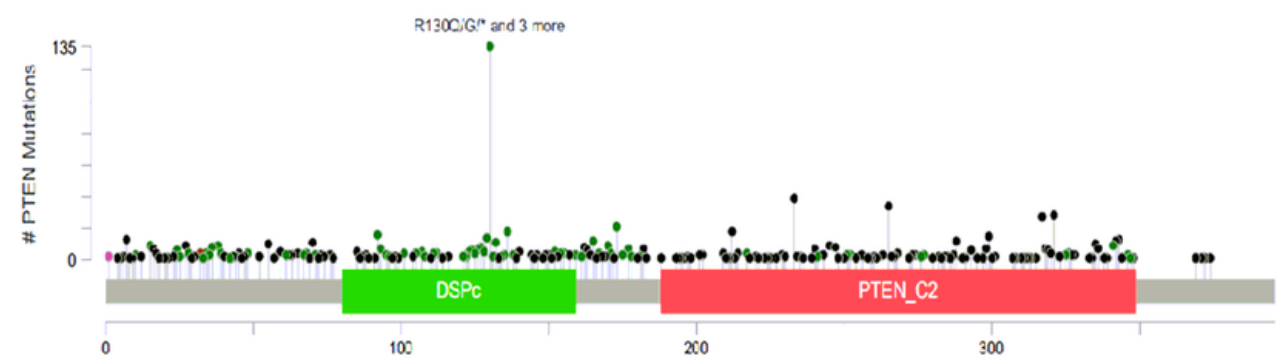

d

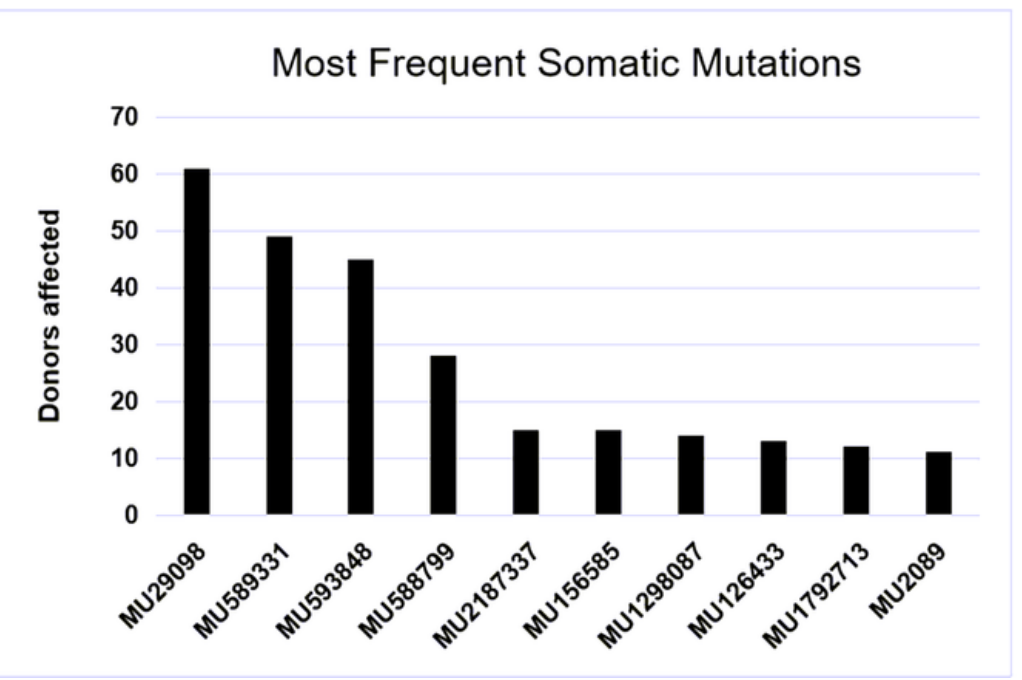

Figure 9

PTEN mutation status and prognosis analysis of HCC patients with PTEN mutation. Notes: (a) The mutation type of PTEN in HCC was assesses by the cBioPortal database. (b) Analysis of disease-free survival rate of HCC patients with PTEN mutation. (c) The frequency and location of PTEN were analyzed by using cBioPortal and ICGC databases. (d) Ranking of the effects of PTEN mutations. Abbreviations: HCC, hepatocellular carcinoma. 
a

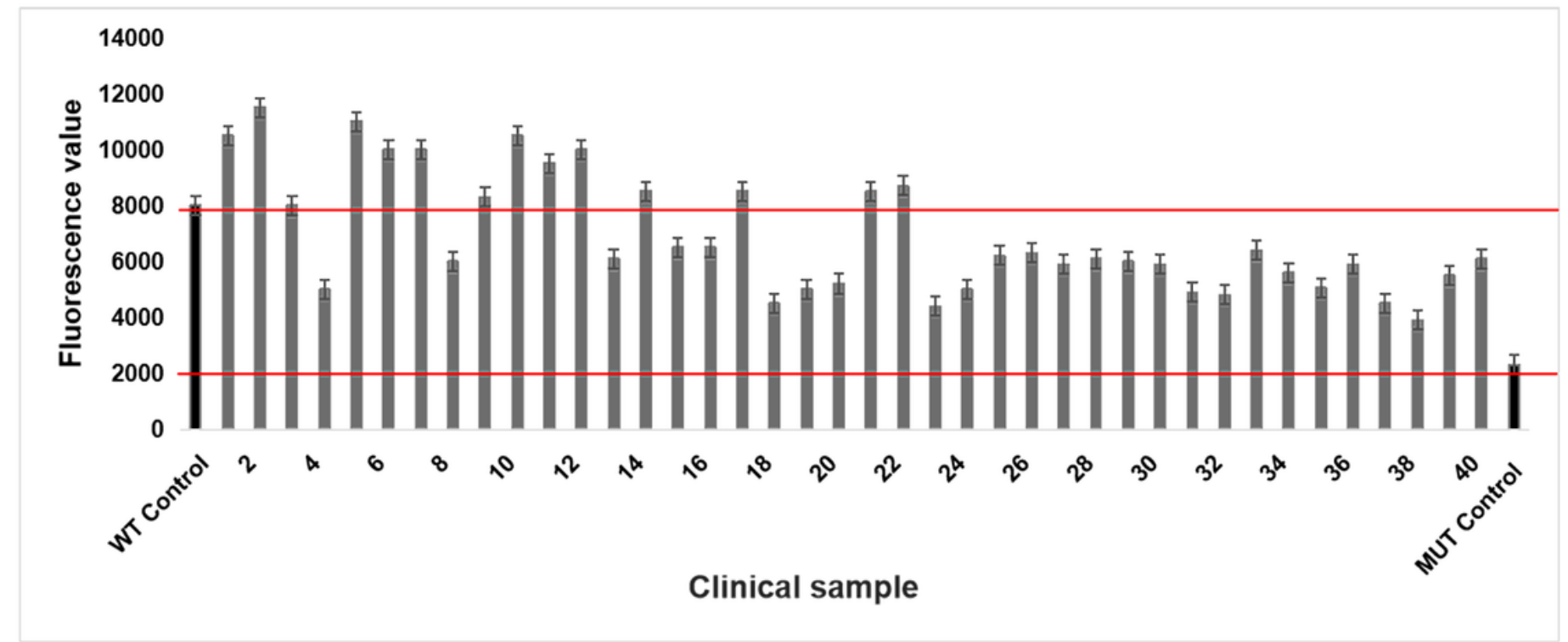

b

R130Q WT

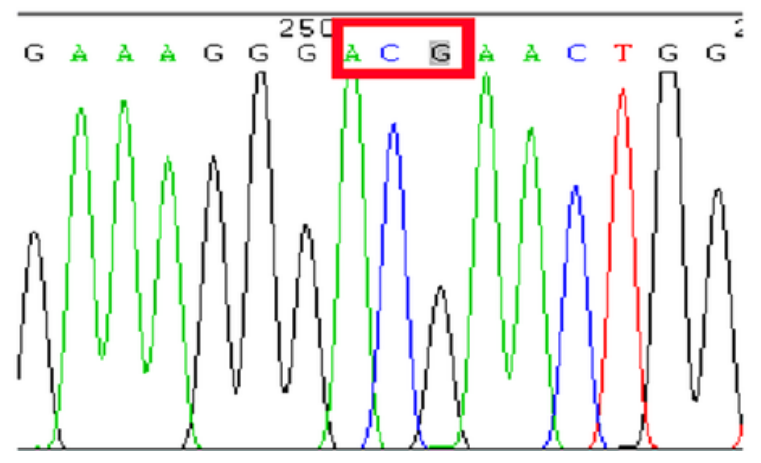

R130Q MUT

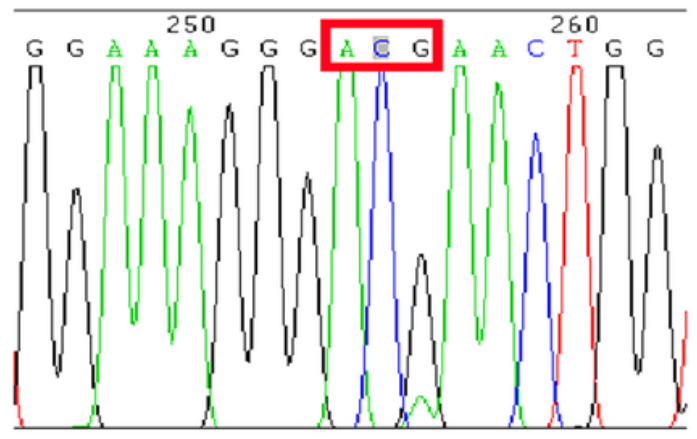

Figure 10

40 clinical samples were detected by CRISPR-cas12a. Notes: (a) Fluorescence values of 40 HCC clinical samples were detected by using CRISPR-cas12a. (b) 40 HCC clinical samples mutations were detected by Sanger sequencing. Abbreviations: HCC, hepatocellular carcinoma. 